JLAB-PHY-01-19

\title{
SPIN PHYSICS AT JLAB IN THE RESONANCE REGION
}

\author{
VOLKER D. BURKERT \\ Jefferson Lab, 12000 Jefferson Avenue, Newport News, VA23606
}

I will discuss recent results from Jefferson Lab on the measurement of inclusive spin structure functions in the nucleon resonance region using polarized ammonia $\mathrm{NH}_{3}$ and polarized ${ }^{3} \mathrm{He}$ targets. Preliminary results on the first moment of $g_{1}\left(x, Q^{2}\right)$ for protons, and the generalized Gerasimov-Drell-Hearn integral for neutrons are presented. In addition, first double polarization data on exclusive electroproduction of $\pi^{+}$from polarized protons will be discussed.

\section{INTRODUCTION}

Since the mid 1970's measurements of polarized structure functions in lepton nucleon scattering have been a focus of nucleon structure physics at high energy laboratories. One of the surprising findings was that less than $25 \%$ of the nucleon spin is accounted for by the spin of quarks [1]. At asymptotic momentum and energy transfers the Bjorken sum rule [4] relates the proton-neutron difference of the first moment $\Gamma_{1}=\int g_{1}(x) d x$ to the weak axial coupling constant $g_{A}$ :

$$
\Gamma_{1}^{p}-\Gamma_{1}^{n}=\frac{1}{6} g_{A}
$$

This sum rule has been evolved in perturbative QCD (pQCD) to finite $Q^{2}$, and has been verified experimentally at the $5 \%$ level. However, the fact, that only a small fraction of the nucleon spin can be directly attributed to the quark spin is in contrast to quark model expectations, and shows that we are far from having a realistic picture of the intrinsic structure of the nucleon. Moreover, the nucleon structure has hardly been explored in the regime of strong interaction which is the true domain of QCD. Our understanding of nucleon structure is incomplete if the nucleon is not probed and fundamentally described at both large and medium distance scales. This is the domain where current experiments at JLab have their greatest impact.

The Gerasimov-Drell-Hearn (GDH) sum rule[2, 3] relates the differences in the helicitydependent total photoabsorption cross sections to the anomalous magnetic moment $\kappa^{2}$ of the target

$$
\frac{M^{2}}{8 \pi^{2} \alpha} \int_{\nu_{0}}^{\infty} \frac{\sigma_{1 / 2}(\nu)-\sigma_{3 / 2}(\nu)}{\nu} d \nu=-\frac{1}{4} \kappa^{2}
$$

where $\nu_{0}$ is the photon energy at pion threshold, and $\mathrm{M}$ is the nucleon mass. This sum rule has been studied for photon energies up to $850 \mathrm{MeV}$ [5], and is currently being tested 

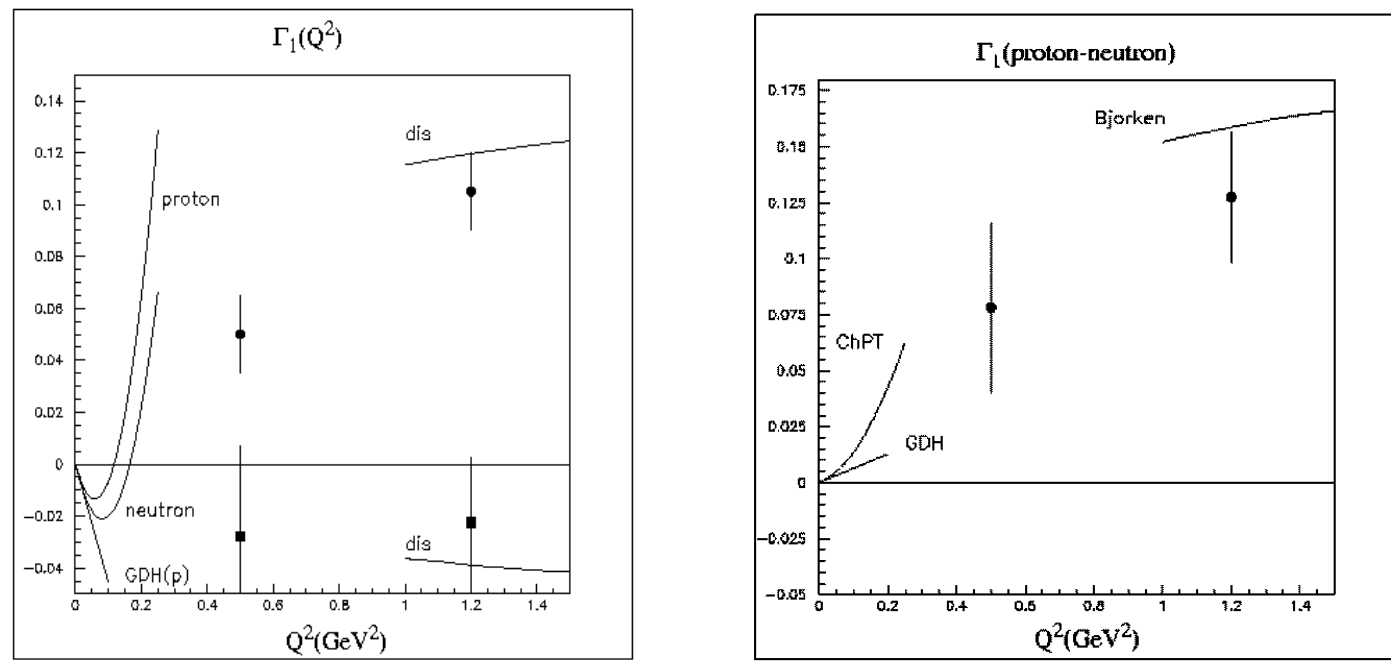

Figure 1. First moments of the spin structure function $g_{1}\left(x, Q^{2}\right)$ for the proton and neutron (left), and for the proton-neutron difference (right). The curves above $Q^{2}=1 \mathrm{GeV}^{2}$ are pQCD evolutions of the measured $\Gamma_{1}$ for proton and neutron, and the pQCD evolution for the Bjorken sum rule, respectively. The straight lines near $Q^{2}=0$ are the slope given by the GDH sum rule. The curves at small $Q^{2}$ represent the NLO HBChPT results.

up to more than $2 \mathrm{GeV}$ at ELSA [6]. A rigorous extension of this sum rule to finite $Q^{2}$ has recently been introduced [7]. This makes a measurement of the $Q^{2}$-dependence of (2) very interesting. It will tell us at what distance scale pQCD corrections and higher twist expansions will break down, and physics of confinement will dominate. It will also allow us to evaluate where resonances give important, even dominant contributions to the first moment 10,11], as well as to the higher $x$-moments of the spin structure function $g_{1}\left(x, Q^{2}\right)$. These contributions need to be determined experimentally and calculated in QCD. The helicity structure of some resonances is changing rapidly with the distance scale probed [14. Accurate measurements will allow stringent tests of nucleon structure models. The well known "duality" between the deep inelastic regime and the resonance regime observed for the unpolarized structure function $F_{1}\left(x, Q^{2}\right)$ needs to be explored for the spin structure function $g_{1}\left(x, Q^{2}\right)$. This will shed new light on this phenomenon which is not well understood. It is only with a concerted effort of precise experiments and new approaches in theory that we will be able to understand nucleon structure from the smallest to the largest distances. It is one of the goals of experiments at JLab to provide the basis for such an endeavor. The first experiments have been completed on polarized hydrogen $\left(\mathrm{NH}_{3}\right)$ and deuterium $\left(\mathrm{ND}_{3}\right)$ targets, and on polarized ${ }^{3} \mathrm{He}$.

\section{EXPECTATIONS FOR $\Gamma_{1}\left(Q^{2}\right)$}

The inclusive doubly polarized cross section can be written as:

$$
\frac{1}{\Gamma_{T}} \frac{d \sigma}{d \Omega d E^{\prime}}=\sigma_{T}+\epsilon \sigma_{L}+P_{e} P_{t}\left[\sqrt{1-\epsilon^{2}} A_{1} \sigma_{T} \cos \psi+\sqrt{2 \epsilon(1+\epsilon)} A_{2} \sigma_{T} \sin \psi\right]
$$




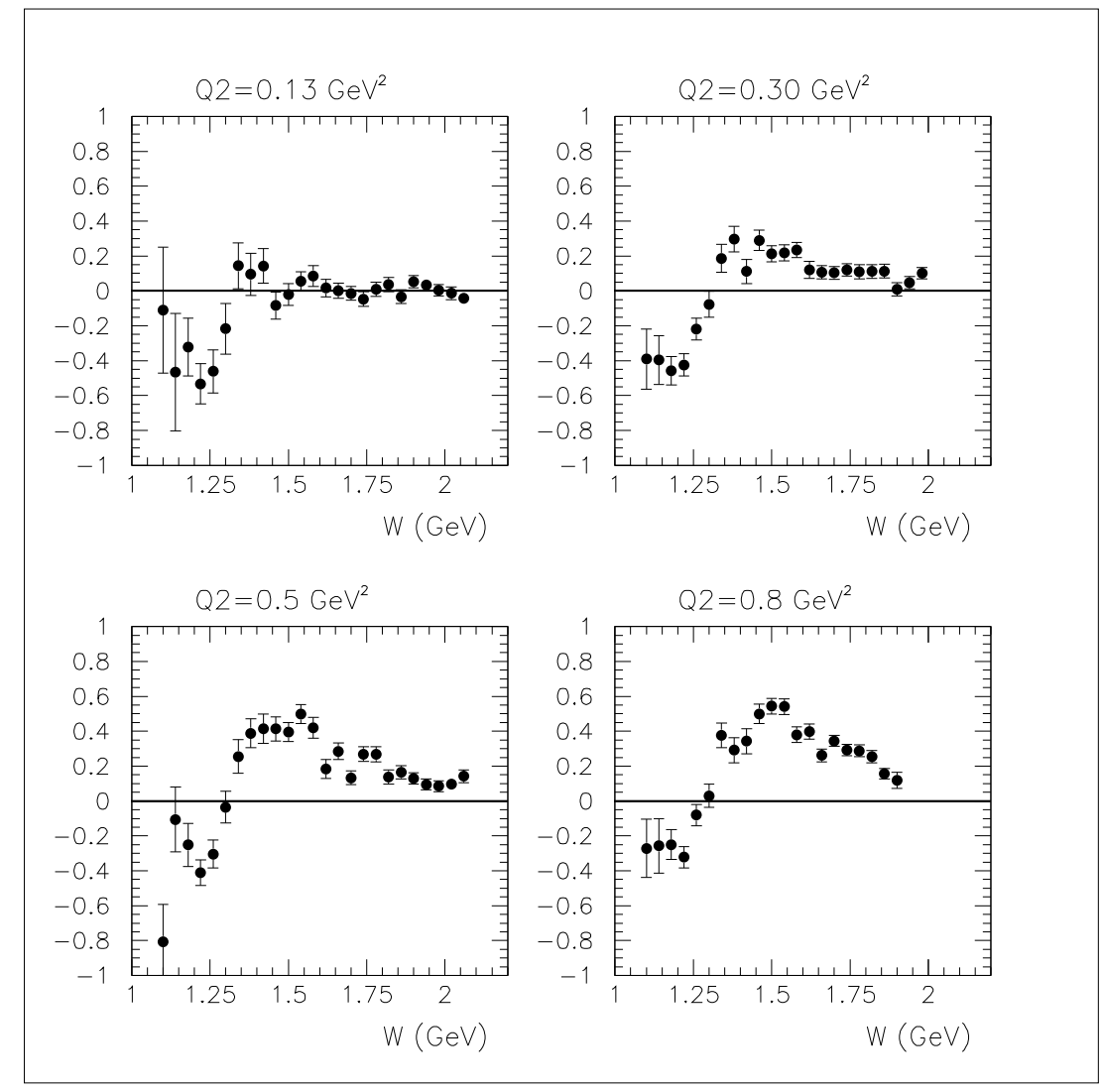

Figure 2. Asymmetry $A_{1}+\eta A_{2}$ for protons. The panels show the results for different $Q^{2}$ values.

where $A_{1}$ and $A_{2}$ are the spin-dependent asymmetries, $\psi$ is the angle between the nucleon polarization vector and the $\vec{q}$ vector, $\epsilon$ the polarization parameter of the virtual photon, and $\sigma_{T}$ and $\sigma_{L}$ are the total absorption cross sections for transverse and longitudinal virtual photons. Experiments usually measure the asymmetry

$$
A_{\text {exp }}=P_{e} P_{t} D \frac{A_{1}+\eta A_{2}}{1+\epsilon R}
$$

where $\mathrm{D}$ is a kinematical factor describing the polarization transfer from the electron to the photon. The asymmetries $A_{1}$ and $A_{2}$ are related to the spin structure function $g_{1}$ by

$$
g_{1}\left(x, Q^{2}\right)=\frac{\tau}{1+\tau}\left[A_{1}+\frac{1}{\sqrt{\tau}} A_{2}\right] F_{1}\left(x, Q^{2}\right)
$$

where $F_{1}$ is the usual unpolarized structure function.

The GDH sum rule defines the slope of $\Gamma_{1}\left(Q^{2}=0\right)$, where we exclude the elastic contribution at $\mathrm{x}=1$ :

$$
2 M^{2} \frac{d \Gamma_{1}}{d Q^{2}}\left(Q^{2} \rightarrow 0\right)=-\frac{1}{4} \kappa^{2}
$$

The GDH and Bjorken sum rules provide constraints at the kinematical endpoints $Q^{2}=0$ and $Q^{2} \rightarrow \infty$. The evolution of the Bjorken sum rule to finite values of $Q^{2}$ using pQCD and the twist expansion allow to connect experimental values measured at finite 

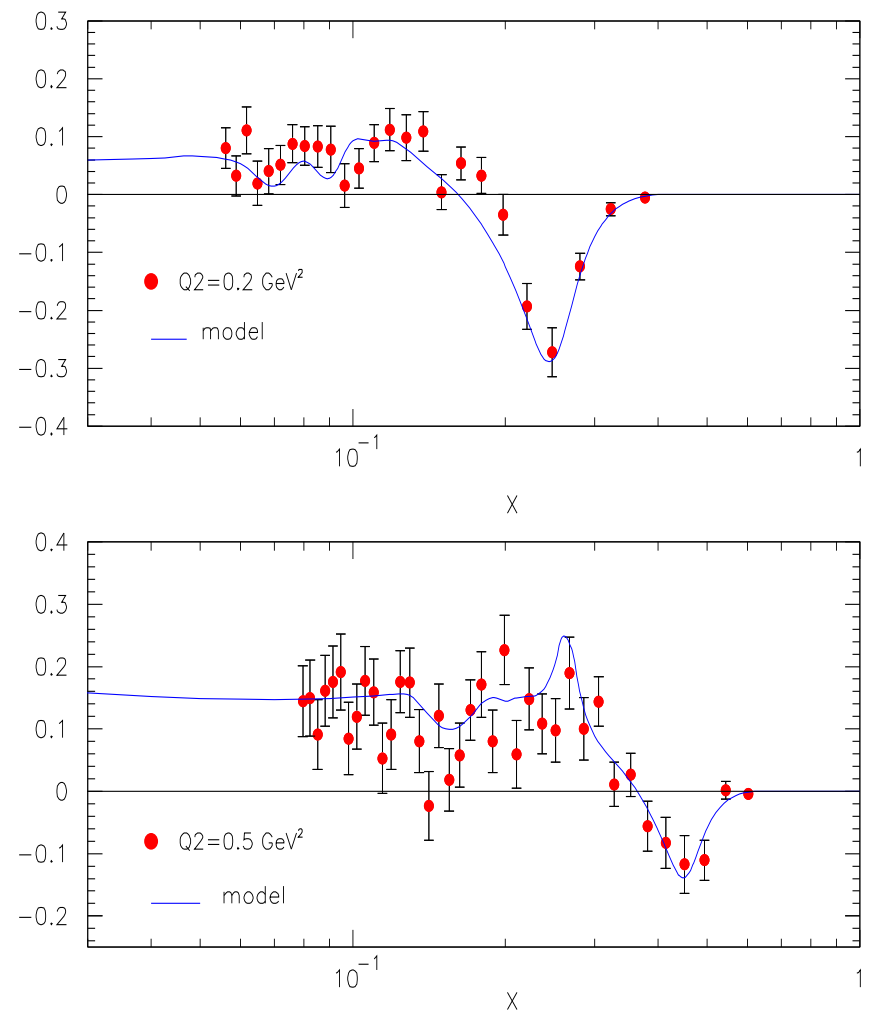

Figure 3. Spin structure function $g_{1}\left(x, Q^{2}\right)$ for the proton. The curve labeled "model" is used for radiative corrections, and to extrapolate to $x=0$ for the evaluation of $\Gamma_{1}$.

$Q^{2}$ to the endpoints. Heavy Baryon Chiral Perturbation Theory (HBChPT) has been proposed as a tool to evolve the GDH sum rule to $Q^{2} \neq 0$, possibly to $Q^{2}=0.1 \mathrm{GeV}^{2}$, and to use the twist expansion down to $Q^{2}=0.5 \mathrm{GeV}^{2}$ [8]. The challenge of nucleon structure physics would be to bridge the remaining gap from $Q^{2}=0.1-0.5 \mathrm{GeV}^{2}$. Lattice QCD may play an important role in describing resonance contributions to the moments of spin structure functions. If successful this would mark the first time that a quantity of nucleon structure physics is described from small to large distances within fundamental theory, a worthwhile goal!

Using just the constraints given by the two endpoint sum rules we may already get a qualitative picture on $\Gamma_{1}^{p}\left(Q^{2}\right)$ and $\Gamma_{1}^{n}\left(Q^{2}\right)$. There is no sum rule for the proton and neutron separately that has been verified. However, experiments have determined the asymptotic limit with sufficient confidence for the proton and the neutron. At large $Q^{2}$, $\Gamma_{1}$ is expected to approach this limit following the pQCD evolution from finite values of $Q^{2}$. At small $Q^{2}, \Gamma_{1}$ must approach zero with a slope given by the GDH sum rule (assuming the sum rule will be verified). The current situation is depicted in Figure 1, where also the next-to-leading HBChPT evolution at small $Q^{2}$ and the pQCD evolution to order $\alpha_{s}^{3}$ at high $Q^{2}$ are shown.

As the slope at $Q^{2}=0$ is $<0, \Gamma_{1}^{p}$ must change sign at some value of $Q^{2}<0.5 \mathrm{GeV}^{2}$. We note that the HBChPT NLO evolution [12] cannot give a good description of the trend shown by the existing data, for $Q^{2}>0.1 \mathrm{GeV}^{2}$. However, for the proton-neutron difference the situation is quite different [13]; the HBChPT curve describes the general trend of the data quite well, and over a significantly larger range in $Q^{2}$ than for proton 


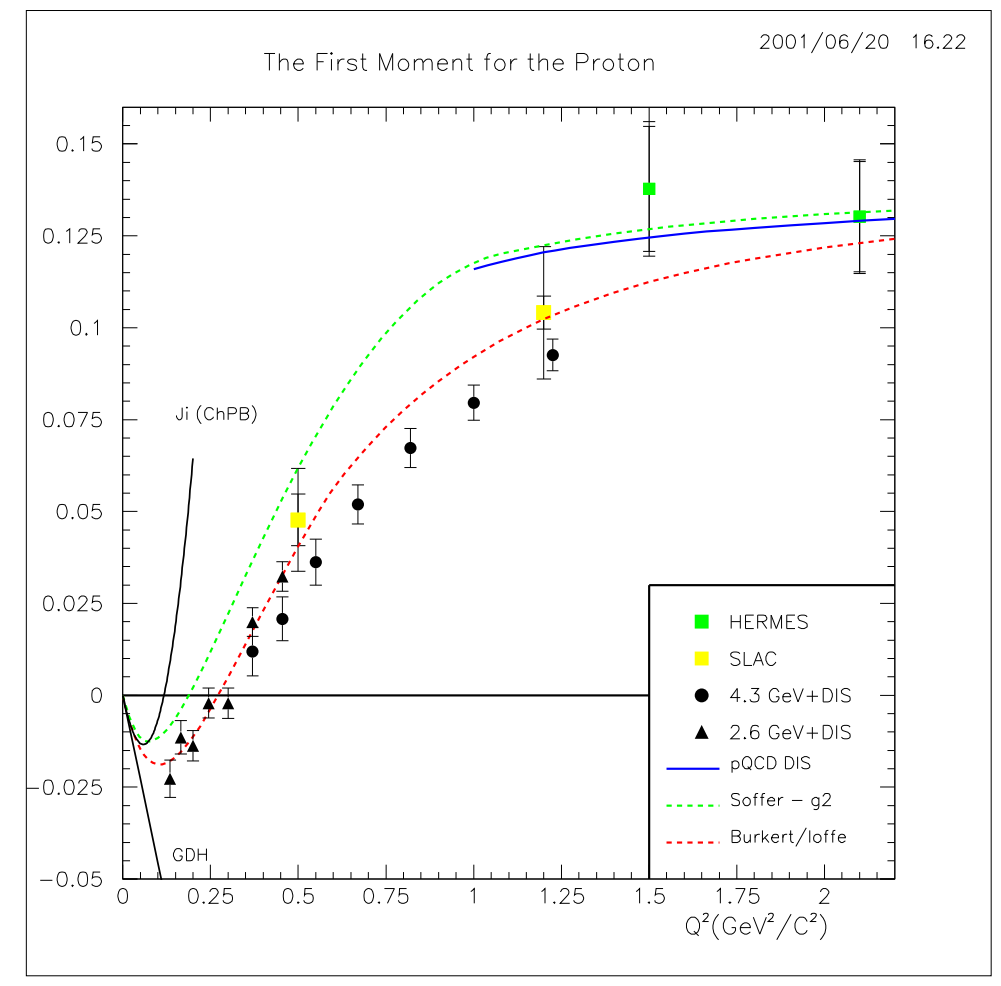

Figure 4. First moment $\Gamma_{1}\left(Q^{2}\right)$ for the proton. The black symbols are preliminary results from CLAS. Data from SLAC and HERMES are shown for comparison

and neutron separately.

\section{PRELIMINARY RESULTS FOR PROTONS AND NEUTRONS.}

Inclusive double polarization experiments have been carried out on polarized hydrogen [15], using $\mathrm{NH}_{3}$ as polarized target material.

In Figure 2 the asymmetry $A_{1}+\eta A_{2}$ is shown for various bins at low $Q^{2}$. In the lowest $Q^{2}$ bin the asymmetry is dominated by the excitation of the $\Delta(1232)$. At higher $Q^{2}$ the asymmetry in the $\Delta(1232)$ region remains negative, however, at higher $\mathrm{W}$ it quickly becomes positive and large, reaching peak values of about 0.6 at $Q^{2}=0.8 \mathrm{GeV}^{2}$ and $\mathrm{W}=1.5 \mathrm{GeV}$. Evaluations of resonance contributions show that this is largely driven by the $S_{11}(1535) A_{1 / 2}$ amplitude, and by the rapidly changing helicity structure of the strong $D_{13}(1520)$ state. The latter resonance is known to have a dominant $A_{3 / 2}$ amplitude at the photon point, but is rapidly changing to $A_{1 / 2}$ dominance for $Q^{2}>0.5 \mathrm{GeV}^{2}$.

Using a parametrization of the world data on $F_{1}\left(x, Q^{2}\right)$ and $A_{2}\left(x, Q^{2}\right)$ we can extract $g_{1}\left(x, Q^{2}\right)$ from (5). Examples of $g_{1}\left(x, Q^{2}\right)$ are shown in Figure 3. The main feature at low $Q^{2}$ is due to the negative contribution of the $\Delta(1232)$ resonance. The graphs also show a model parametrization of $g_{1}\left(x, Q^{2}\right)$ which was used to extrapolate to $x \rightarrow 0$. The extrapolation is needed to evaluate the first moment $\Gamma_{1}\left(Q^{2}\right)$.

The results for $\Gamma_{1}^{p}\left(Q^{2}\right)$ are shown in Figure 4 . The characteristic feature is the strong $Q^{2}$ dependence for $Q^{2}<1 \mathrm{GeV}^{2}$, with a zero crossing near $Q^{2}=0.3 \mathrm{GeV}^{2}$. This result is preliminary, and the final results may change within the systematic uncertainties. However, the qualitative features of the data will not change. 


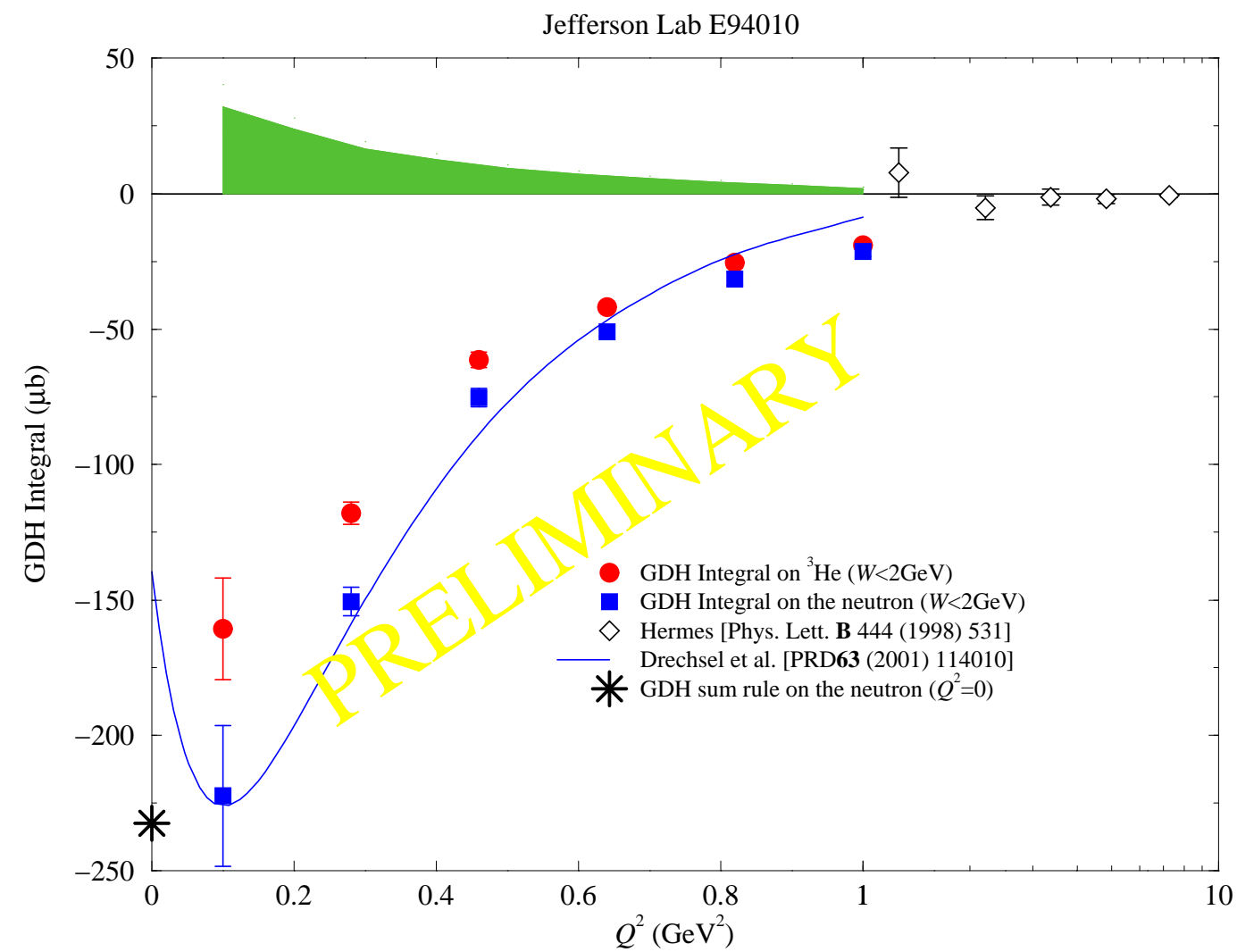

Figure 5. Generalized GDH integral for ${ }^{3} \mathrm{He}$ and neutron. The dark band indicates the systematic errors.

Measurements on $\mathrm{ND}_{3}$ have been carried out in CLAS [16], and on ${ }^{3} \mathrm{He}$ in Hall A [17] to measure the corresponding integrals for the neutron. Data were taken with the JLab Hall A spectrometers using a polarized ${ }^{3} \mathrm{He}$ target. Since the data were taken at fixed scattering angle, $Q^{2}$ and $\nu$ are correlated. Cross sections at fixed $Q^{2}$ are determined by an interpolation between measurements at different beam energies. Both longitudinal and transverse settings of the target polarization were used. Therefore no assumptions about $A_{2}$ are necessary in this case. The GDH integral for ${ }^{3} \mathrm{He}$ and for neutrons is shown in Figure 5. The integral is evaluated over the region from pion threshold (on a free neutron) to $\mathrm{W}=2 \mathrm{GeV}$, to cover the resonance region only. Corrections for nuclear effects were made based on a prescription by Ciofi degli Atti [18].

The GDH integral for the neutron shows a $Q^{2}$ behavior that seems to smoothly approach the GDH sum rule value. However, the data are also in reasonable agreement with the model curve, which indicates a rapid departure away from the sum rule value at very small $Q^{2}$. Data at smaller $Q^{2}$ and for real photons should clarify the situation.

\section{SPIN RESPONSES IN EXCLUSIVE CHANNELS}

The CLAS detector with its large acceptance and good resolution offers the possibility of studying spin observables for exclusive channels. All single pion channels are currently being studied. These measurements give information on the isospin dependence of the helicity structure in pion production. Since many resonances couple strongly to the $N \pi$ 
channel we also obtain information on the helicity structure of resonance excitations. I discuss briefly the $n \pi^{+}$channel where final results have been obtained. For details see the talk presented by R. DeVita in the parallel session [19].

Figure 6 shows $A_{1}+\eta A_{2}$ for an average $Q^{2}=0.6 \mathrm{GeV}^{2}$. This channel has a large non-resonant contribution due to the t-channel pion pole term. Resonance production is, therefore, somewhat masked. This is particularly evident in the region of the $\Delta(1232)$ where the measured asymmetry is small. This is in contrast to the previously discussed inclusive reaction, and also to the expected asymmetry $A_{1}=-0.5$ for a pure magnetic dipole transition such as the $\Delta(1232)$.

Above the $\Delta$ mass region, the asymmetry is large and positive similar to the inclusive results. Models that include s-channel resonance excitations make predictions for the $Q^{2}$-dependence of $A_{1}+\eta A_{2}$ based on the analysis of angular distributions of unpolarized differential cross sections 20,21. While for the $\Delta(1232)$ a rather small asymmetry, with rather weak $Q^{2}$ dependence, is predicted, the higher mass regions should have a large asymmetry with a strong tendency the towards maximum value of +1 . Figure 6 shows different ranges in $\mathrm{W}$ corresponding to different resonance regions. The asymmetry in the region of the Roper resonance is nearly maximum and dominated by non-resonant contributions. The region near $\mathrm{W}=1.5 \mathrm{GeV}$ is dominated by the $S_{11}(1535)$ and $D_{13}(1535)$ states, and model parametrizations predict large and positive asymmetries rising with $Q^{2}$. This is qualitatively seen in the data. The largest discrepancy between the data and the model calculations is seen in the 3rd resonance region. While the trend of the data is reproduced, the resonance contributions are significantly underestimated.

We also note that $A_{1}+\eta A_{2}$ in the resonance region is significantly larger than in the deep inelastic domain.

\section{Conclusions and Outlook}

First measurements of double polarization asymmetries have been carried out at Jefferson Lab in a range of $Q^{2}$ not covered in high energy experiments. The results show large contributions from resonance excitations with a rapidly changing helicity structure. The first moment $\Gamma_{1}^{p}\left(Q^{2}\right)$ of the spin structure function $g_{1}\left(x, Q^{2}\right)$ has been extracted. It shows a dramatic change with $Q^{2}$, including a sign change near $Q^{2}=0.3 \mathrm{GeV}$. Qualitatively, this marks the transition from the domain of single parton physics to the domain of resonance excitations and hadronic degrees of freedom.

For the first time beam-target asymmetries have been measured in exclusive $\pi^{+}$electroproduction. Large asymmetries are seen in the resonance region. With increasing $Q^{2}$ they tend to rapidly approach the limit where helicity $1 / 2$ production dominates the process.

New data have been taken both on hydrogen and deuterium with nearly 10 times more statistics, and higher target polarizations, and over a larger range of energies from 1.6 $\mathrm{GeV}$ to $5.75 \mathrm{GeV}$. These data cover a $Q^{2}$ range from 0.05 to $2.5 \mathrm{GeV}^{2}$, and a larger part of the deep inelastic regime than our previous data. This will allow to reduce the systematic uncertainties related to the extrapolation to $x=0$. The new data will also give much better sensitivity to resonance production in a large number of exclusive channels. Finally, at the higher energies, we will be able to study single spin asymmetries in various inclusive and exclusive reactions. 


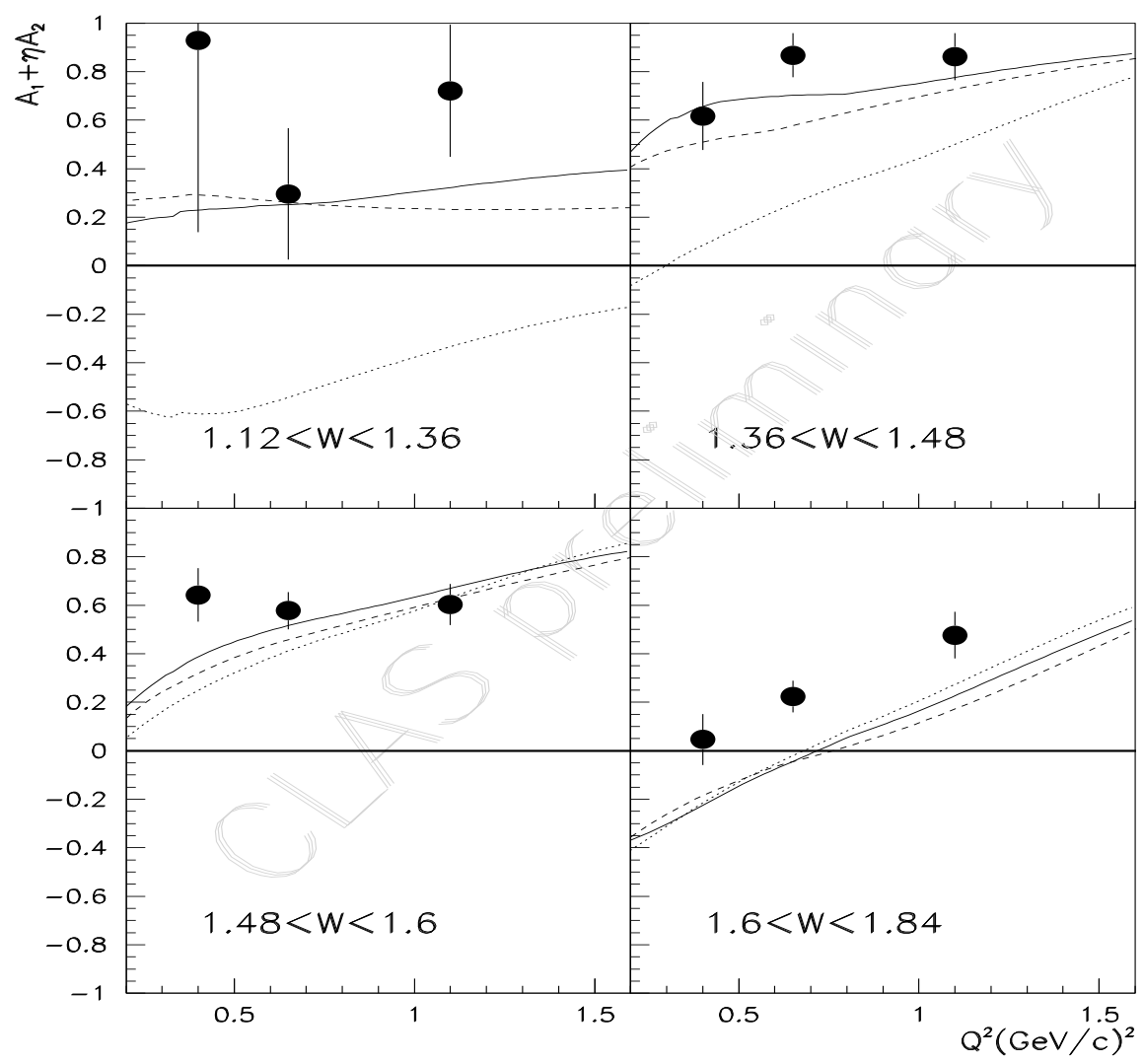

Figure 6. Helicity asymmetry $A_{1}+\eta A_{2}$ for the process $\vec{p}\left(\vec{e}, e^{\prime} \pi^{+}\right) n$. The curves are from parametrizations of s-channel resonances and non-resonant Born term contributions to single pion production. Solid lines are from the full calculation from AO [20]. Dotted lines are from AO for resonances only. Dashed lines are the full calculation from MAID 21]. The various panels are for different ranges in the hadronic mass spectrum.

There is also a program underway in JLab Hall A to measure the GDH integral for neutrons down to extremely small $Q^{2}$ values, near the real photons point, and to measure the asymmetry $A_{1}\left(x, Q^{2}\right)$ for the neutron at high x.

The Southeastern University Research Association (SURA) operates JLab for the U.S. Department of Energy under Contract No. DE-AC05-84ER40150.

\section{REFERENCES}

1. For a recent review see: B.W. Filippone, Xiangdong Ji; hep-ph/0101224 (2001)

2. S.B. Gerasimov; Sov. J. Nucl. Phys. 2, 430 (1966)

3. S.D. Drell and A.C. Hearn, Phys. Rev. Lett.16 (1966) 908

4. J.D. Bjorken, Phys. Rev. 179, 1547 (1969)

5. J. Ahrens et al., hep-ex/0105089 (2001)

6. G. Anton, in: Few Body Systems, Springer, pg. 177 (1998), Proceedings of the Workshop on $\mathrm{N}^{*}$ Physics and Nonperturbative QCD, eds. S. Simula, B, Saghai, N, Mukhopadhyay, V. Burkert, Trento, Italy. 
7. X. Ji, J. Osborne, J.Phys.G27:127,2001

8. X. Ji, Proceedings NSTAR2000 conference, Jefferson Lab, Feb. 16-19, 2000. Eds. V.D. Burkert, L. Elouadrhiri, J.J. Kelly, R. C. Minehart, World Scientific.

9. K. Abe et al., Phys. Rev. D58, 2003 (1998)

10. V. Burkert and Zh. Li, Phys. Rev. D47,46(1993)

11. V. Burkert and B. Ioffe, Phys. Letts. B296 (1992) 223; J. Exp. Theo. Phys.78, 619(1994)

12. X. Ji, C.W. Kao, J. Osborne, Phys.Lett.B472:1-4,2000

13. V. Burkert, Phys. Rev. D63, 97904(2001)

14. V. D. Burkert, Czech. Journal of Physics,Vol. 46, 627, 1996.

15. V. Burkert, D. Crabb, R. Minehart, et al., JLab experiment 91-023.

16. S. Kuhn, G. Dodge, M. Taiuti, et al., JLab experiment 93-009

17. Z.E. Meziani et al., experiment E94-010

18. C. Ciofi degli Atti, S. Scopetta, Phys.Lett.B404:223-229,1997

19. R. DeVita, talk at this conference

20. AO code, see: V.D. Burkert and Zh. Li, PRD46,47 (1993); R. DeVita, private communications.

21. D. Drechsel et al., Nucl. Phys. A645, 145 (1999) 